Check for updates

Cite this: RSC Adv., 2019, 9, 32141

Received 22nd August 2019

Accepted 19th September 2019

DOI: $10.1039 / c 9 r a 06622 e$

rsc.li/rsc-advances

\section{High-throughput metabolomics for evaluating the efficacy and discovering the metabolic mechanism of Luozhen capsules from the excessive liver-fire syndrome of hypertension $\uparrow$}

\author{
Xi-jun Wang, (D)*ab Xin Gao, ${ }^{\text {ab }}$ Ai-hua Zhang, ${ }^{\text {ab }}$ Fang-fang Wu, ${ }^{\text {ab }}$ Guang-li Yan ${ }^{\text {ab }}$ \\ and Hui Sun ${ }^{\text {ab }}$
}

Essential hypertension (EH) is a chronic disease characterized by a variety of causes of elevated systemic arterial pressure, which often causes functional or organic damage to important organs such as the heart, brain, and kidney. Hypertension of excessive liver-fire syndrome is a type of classification for young people with essential hypertension. The disease is slower in its onset and its symptoms are more ambiguous, and thus its pathogenesis is complicated and still unclear. In this study, aconite, dried ginger and cinnamon extracts were combined with L-NAME to establish a model of excessive liver-fire hypertension. Blood pressure (systolic blood pressure), ANGIl, NE and 5-HT were used as evaluation indicators to establish the model. Urinary metabolomics based on ultra-high performance liquid chromatography coupled with quadruple time-of-flight mass spectrometry was used to characterize the metabolic changes and potential biomarkers in modeled rats. Compared to the treatment group, 32 potential biomarkers were initially identified in the model using multivariate statistical analysis involving 11 metabolic pathways. After oral administration of Luozhen capsules, eight biomarkers that can be adjusted in high, medium and low doses of Luozhen capsules in urine were preliminarily determined, mainly involving two metabolic pathways of amino acid metabolism and lipid metabolism. In conclusion, this study explored the metabolomic changes in rats with hypertension of liver-fire hyperactivity syndrome and the post-dose metabolomics, determined the relevant biomarker groups, and clarified the metabonomic connotation of Luozhen capsules in the treatment of liver-fire excessive type hypertension.

\section{Introduction}

Essential hypertension is one of the high risk factors for cardiovascular and cerebrovascular diseases, such as heart failure, myocardial infarction and stroke, and has become one the main causes of global disease. With the increasing living standards, high blood pressure is increasing. In 1975, the number of patients with global essential hypertension was 590 million (prevalence rate was 14.5\%). By 2015, it had grown to 1.13 billion (prevalence rate was 15.3\%). The current growth rate is expected to increase the number of patients with

${ }^{a}$ National Engineering Laboratory for the Development of Southwestern Endangered Medicinal Materials, Guangxi Botanical Garden of Medicinal Plant, Nanning, Guangxi, China

${ }^{b}$ National Chinmedomics Research Center, Sino-America Chinmedomics Technology Collaboration Center, National TCM Key Laboratory of Serum Pharmacochemistry, Laboratory of Metabolomics, Department of Pharmaceutical Analysis, Heilongjiang University of Chinese Medicine, Heping Road 24, Harbin 150040, China. E-mail: xijunwangls@126.com; Fax: +86-451-82110818; Tel: +86-451-82110818

$\dagger$ Electronic supplementary information (ESI) available. See DOI: 10.1039/c9ra06622e hypertension worldwide to 1.56 billion by $2025 .^{1-5}$ If its treatment is not timely, long-term illness will lead to complications, such as coronary heart disease, diabetes, heart failure, and kidney disease, which will seriously affect human health and life and result in a huge economic burden to families and society. ${ }^{6}$ In recent years, domestic and foreign scholars have carried out in-depth research on the regulation mechanism of the occurrence and development of hypertension, and it has been found that there are many possible mechanisms for its development. The mechanism of Western medical tradition is the renin-angiotensin-aldosterone system (RAAS) and the sympathetic nervous system (SNS). Two major systems are involved in regulating the salt-water balance and cardiovascular function disorders. ${ }^{7}$ Some scholars have speculated that hypertension may be a disease dominated by autoimmunity, such as the immune regulation involved in macrophages. ${ }^{8-10}$ In addition, gene regulation, central nervous system dysfunction, kidney damage, etc. are also mentioned. However, current treatments aimed at limiting the effects of RAAS or SNS on blood pressure have a high failure rate, and thus the clarification of essential hypertension mechanisms must be a priority. 
In 2017, the American College of Cardiology and the American Heart Association further emphasized the importance of discovering the mechanisms of essential hypertension. ${ }^{\mathbf{1 1}}$ Chinese medicine believes that hypertension is a continuous pathophysiological process. According to the pathological characteristics of different stages, different syndromes are distinguished, and thus the development of syndromes and diseases can be integrated with each other. Presently, due to the different clinical manifestations of hypertension, there are different types of syndromes, which are generally divided into four types: phlegm, yin and yang, yin deficiency and impotence, and liver qi stagnation. Under certain environmental conditions, these four syndromes can be transformed into each other. In general, most are hepatic and turbid, which are converted to yin and yang and yin deficiency. Among the four types of syndromes, liver stagnation is a common syndrome of clinical liver disease with the highest incidence. Therefore, the study of this syndrome is of great significance for the prevention and treatment of hypertension. ${ }^{\mathbf{1 2 - 1 5}}$

Experimental teams have been working on metabolomics research, which has now formed its own characteristics. The doctor's disease is matched with the chinese medicine syndrome, and the syndrome type is classified. After the classification, the cause of the disease or the stage of the disease can be more accurately explained. Wu et al. used UPLCQ-TOF-MS combined with the pattern recognition method for urinary metabolomics research to evaluate the changes in metabolic profiles, identify biomarkers, and clarify that Guizhi Fuling Wan (GFW) treats endometriosis of cold coagulation under the premise of determining its effective treatment. The mechanism of coagulation and blood stasis (ECB) laid the foundation for further study of the pharmacodynamic mechanism of GFW. ${ }^{16}$ Wang et al. established a model of kidney yin deficiency and kidney yang deficiency. Based on the establishment of the syndrome model, the small molecule metabolites in body fluids were found to be used for similar metabolic profiles and biomarkers. ${ }^{17}$ Biomarkers have gradually become the main basis for accurate diagnosis, which is a bridge connecting basic research and clinical diagnosis and treatment of precision medicine. Based on this foundation, we continued to study the classification of hypertensive liver disease. Firstly, we evaluated the established hypertension model. On the premise of the success of the model, metabolite changes in patients with hypertension of excessive liver-fire syndrome were investigated, relevant biomarkers were identified, and disturbed metabolic pathways were discovered to speculate the pathogenesis of hypertension. Urinary metabolomics technology was used to evaluate the efficacy of Luozhen capsules in the treatment of excessive liver-fire syndrome of hypertension and to find the basis for its pharmacological substance.

\section{Experimental}

\subsection{Reagents and materials}

Distilled water was purchased from Watsons (Harbin, China). Methanol and acetonitrile (HPLC grade) were obtained from Merck (Darmstadt, Germany). Formic acid (HPLC grade) was purchased from Tianjin Kemiou Chemical Reagent Co., Ltd. (Tianjin, China). NG-nitro-L-arginine methyl ester was purchased from Bailingwei Technology Co., Ltd. (Beijing, China). Pentobarbital sodium powder was purchased from Shanghai Chemical Reagent Purchasing and Supplying station, China Pharmaceutical Group. (Shanghai, China). NaCl was obtained from Macklin Biochemical Technology Co., Ltd. (Shanghai, China). The Norepinephrine (NE) ELISA kit, 5hydroxytryptamine (5-HT) ELISA kit and the angiotensin (ANG II) ELISA kit were made by R\&D Systems (USA). Luozhen capsules were provided by Wusulijiang Pharmaceutical Co., Ltd. (Harbin, B20020220). Tianma Ganteng granules were purchased from Jiuzhitang Jinding Pharmaceutical (Chengdu, China).

Aconite was derived from the roots of Aconitum carmichaelii Debx, the dried ginger was a piece of the root of Zingiber officinale Rose, and the cinnamon was the dried bark of Cinnamomum cassis Presl. The above medicinal materials were purchased from Beijing Tongrentang (Harbin, China), and were certified by the teachers of the Chinese Medicine Identification Laboratory of Heilongjiang University of Traditional Chinese Medicine. All the herb specimens were kept in the laboratory at Heilongjiang University of Traditional Chinese Medicine.

\subsection{Solution preparation}

2.2.1 Preparation of molding solution. The decoction pieces of aconite, dried ginger and cinnamon (1:1:1) were soaked in 10 times distilled water for $30 \mathrm{~min}$, decocted twice, $30 \mathrm{~min}$ each time, twice. The decoction was concentrated in a liquid containing $1 \mathrm{~g} \mathrm{~mL} \mathrm{~mL}^{-1}$ of crude drug, and L-NAME was added as the model solution.

2.2.2 Preparation of administration solution. First, the appropriate amount of Luozhen capsules was weighed, dissolved in distilled water, and sonicated for $15 \mathrm{~min}$ to obtain the Luozhen capsule solution. The high, medium and low doses of Luozhen capsules were four, two and one times the weight of the human body $\left(0.432 \mathrm{~g} \mathrm{~kg}^{-1} \mathrm{~d}^{-1}, 0.216 \mathrm{~g} \mathrm{~kg}^{-1} \mathrm{~d}^{-1}, 0.108 \mathrm{~g}\right.$ $\mathrm{kg}^{-1} \mathrm{~d}^{-1}$, respectively). Secondly, the appropriate amount of Tianma Gouteng granules was weighed and dissolved in distilled water for $15 \mathrm{~min}$ to obtain a positive control solution. The dose of the positive control drug Tianma Gouteng granules was doubled $\left(1.34 \mathrm{~g} \mathrm{~kg}^{-1} \mathrm{~d}^{-1}\right)$. The dose administered was calculated as the clinical dose taken by a person.

\subsection{Modeling method}

On the 1st to 28 th day of the experiment, except for the K group, the other 5 groups of rats were given the model solution by intragastric administration, and $1 \%$ saline was freely consumed for 28 consecutive days. On the 29th to 56th day of the experiment, the $\mathrm{M}$ group was given distilled water by gavage, in group $\mathrm{Y}, 1.34 \mathrm{~g} \mathrm{~kg}^{-1} \mathrm{~d}^{-1}$ positive control drug solution was administered by gavage, $0.432 \mathrm{~g} \mathrm{~kg}^{-1} \mathrm{~d}^{-1}, 0.216 \mathrm{~g} \mathrm{~kg}^{-1} \mathrm{~d}^{-1}$ and $0.108 \mathrm{~g}$ $\mathrm{kg}^{-1} \mathrm{~d}^{-1}$ Luozhen capsule liquid were administered by intragastric administration in $\mathrm{H}, \mathrm{Z}$ and $\mathrm{L}$ groups, respectively, and the $\mathrm{K}$ group was given distilled water daily for 56 days. The 
amount of gastric perfusion was performed per $100 \mathrm{~g} \mathrm{~mL}^{-1}$ of the body weight of the rats.

\subsection{Experimental design}

Sixty male Wistar rats weighing $200 \mathrm{~g}+20 \mathrm{~g}$ were randomly divided into six groups: control group (C, $n=10)$, model group $(\mathrm{M}, n=10)$, positive control group (Y, $n=10)$, Luozhen capsule high, medium and low dose group $(\mathrm{H}, \mathrm{Z}$, and $\mathrm{L}$, respectively, $n=$ $10)$. On the 1 st to 28 th day of the experiment, except for the $\mathrm{C}$ group, the other 5 groups of rats were modeled according to 2.3, where on the 29th to 56th day of the experiment, the M group was given distilled water by gavage and the $\mathrm{Y}$ group was given $1.34 \mathrm{~g} \mathrm{~kg}^{-1} \mathrm{~d}^{-1}$ positive control drug by gavage. Groups $\mathrm{H}, \mathrm{M}$ and $\mathrm{L}$ were intragastrically administered with $0.432 \mathrm{~g} \mathrm{~kg}^{-1} \mathrm{~d}^{-1}$, $0.216 \mathrm{~g} \mathrm{~kg}^{-1} \mathrm{~d}^{-1}$ and $0.108 \mathrm{~g} \mathrm{~kg}^{-1} \mathrm{~d}^{-1}$ Luozhen capsules and the $\mathrm{C}$ group was given distilled water daily for 56 days. The amount of gastric perfusion was performed per $100 \mathrm{~g} \mathrm{~mL}^{-1}$ of the body weight of the rats. The experimental procedures were approved by the Animal Care and Ethics Committee at Heilongjiang University of Chinese Medicine and all experiments were performed in accordance with the Declaration of Helsinki.

\subsection{Determination of blood pressure}

The blood pressure of the rats was measured every 7 days and continuously recorded from day 0 to day 28 of modeling. The instrument was preheated for $10 \mathrm{~min}$ before the measurement, and the temperature of the heat pump was controlled at $36^{\circ} \mathrm{C}$ to $36.5{ }^{\circ} \mathrm{C}$. The rats were fixed with a fixture, measured 3 times, and the average value was taken.

\subsection{Serum sample collection and pretreatment}

Serum samples were collected from the 6 groups of rats on the 56th day. All animals were fasted for $12 \mathrm{~h}$ before sampling with free access to water, and intraperitoneally injected with $3 \%$ sodium pentobarbital $\left(2 \mathrm{~mL} \mathrm{~kg}{ }^{-1}\right)$ for anesthesia. Blood samples were collected from their hepatic portal vein for $30 \mathrm{~min}$, and then centrifuged for $15 \mathrm{~min}$ at 4 and $4000 \mathrm{rpm}$, and the supernatant was collected and stored at $-80{ }^{\circ} \mathrm{C}$.

\subsection{Biochemical indicator detection}

Serum biochemical indicators (5-HT, NE and ANG II) were measured by spectrophotometry using a microplate reader (PerkinElmer, Microplate Reader-2030, USA) according to the operating manual of the enzymatic kits.

\subsection{Metabonomics analysis}

2.8.1 Conditions of chromatography and mass spectrometry. A Waters ACQUITY UPLC System equipped with a binary solvent delivery system was applied for chromatographic separation analysis. An Acquity UPLCTM HSS T3 column (100 mm $\times 2.1 \mathrm{~mm}, 1.7 \mu \mathrm{m}$ ) (Waters, USA) was applied for the analysis of the samples. The conditions for UPLC were optimized, including column temperature $\left(40{ }^{\circ} \mathrm{C}\right)$, flow rate $(0.40$ $\mathrm{mL} \mathrm{min}^{-1}$ ), and mobile phase [(A) $0.1 \%$ (by volume) formic acid in acetonitrile and (B) $0.1 \%$ (by volume) formic acid in acetonitrile water]. The gradient program for urine metabolism was optimized as shown in Table S1. $\dagger$

A Q-TOF analyzer, electron spray ionization system was applied to collect mass spectrometry data, both in positive and negative ion modes. The specific parameter settings were as follows: positive ion mode mass spectrometry conditions: capillary voltage: $2.6 \mathrm{kV}$; cone hole sampling voltage: $30 \mathrm{~V}$; cone hole extraction voltage: $3.0 \mathrm{~V}$; desolvation gas temperature: $380{ }^{\circ} \mathrm{C}$; and desolvation gas flow: $800 \mathrm{~L} \mathrm{~h}^{-1}$; and negative ion mode mass spectrometry conditions: capillary voltage: $2.0 \mathrm{kV}$; taper hole sampling voltage: $35 \mathrm{~V}$; taper hole extraction voltage: $3.5 \mathrm{~V}$; desolvation gas temperature: $380{ }^{\circ} \mathrm{C}$; and desolvation gas flow rate: $800 \mathrm{~L} \mathrm{~h}^{-1}$. Both positive and negative ion mode scanning methods were full scan, and the quality data collection range was $m / z$ 50-1500.

2.8.2 Data preprocessing and multivariate statistical analysis. QI and MarkerLynx (Waters, U.S.A.) were applied to analyze the metabonomics data. The raw data was dropped in dimension by QI firstly. Then, principal component analysis (PCA) and orthogonal partial least squares discriminate analysis (OPLSDA) were successively applied to distinguish the sample metabolic profiles and determine the difference in metabolites between the different groups.

\section{Results}

\subsection{Evaluation of excessive liver-fire syndrome of hypertension rat model}

3.1.1 Determination of blood pressure. On the 0th day of the blood pressure test, the blood pressure of each group was in the range of 105-110 $\mathrm{mmHg}$. On the 28 th day of the experiment, the blood pressure of the rats in group $\mathrm{C}$ was stable and they exhibited normal growth. The blood pressure of the rats in group $\mathbf{M}$ was always higher than that of the $\mathrm{C}$ group $(P<0.01)$, with the average value increased to $131 \mathrm{mmHg}$ from $109 \mathrm{mmHg}$, and the results are shown in Fig. 1.

3.1.2 Biochemical features. After 28 days of modeling, the levels of ANGII, NE and 5-HT in the serum of group $\mathrm{M}$ were significantly increased compared with that of group $\mathrm{C}(P<0.01)$, and the results are shown in Fig. 2.

3.1.3 The changes of the urine metabolic profile and biomarkers. On the 0th, 14th, and 28th day of the model, urine was supplied with the test solution, and the urine data was obtained according to the analysis conditions of 2.8.1. The peaks

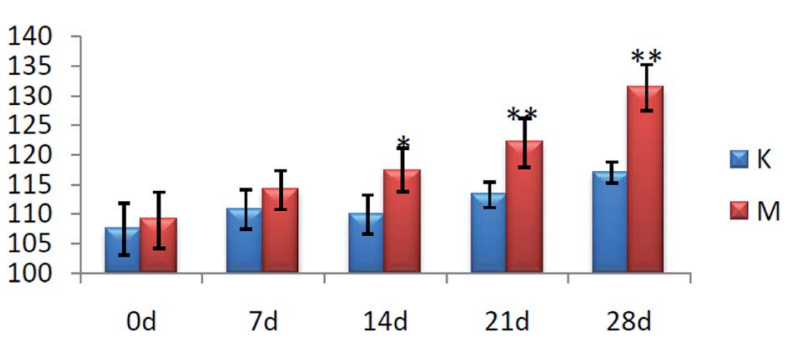

Fig. 1 Blood pressure levels of the rats in each group $(* P<0.05, * * P<$ 0.01 , compared with the control group). K: control group and $M$ : model group. 

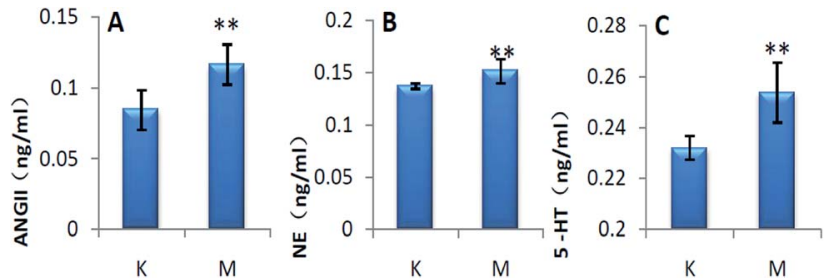

Fig. 2 Biochemical indicators of the rats in each group $(* P<0.05, * * P$ $<0.01$, compared with K group). K: control group, M: model group, A: ANGII, B: NE, and C: 5-HT.

were matched, extracted, and normalized, and the ions were normalized. Subsequently, unsupervised subjective analysis (PCA) was applied to obtain a score plot that reflects the process of the model change, as shown in Fig. 3. As can be seen, on the 14th and 28th day, the data contour gradually moved away from the 0th day, and the 28th day showed complete separation, and the metabolic change was the most significant. The 28th day urine metabolic profile data was input into the Progenesis QI software, and unsupervised subjective analysis (PCA) was performed on all ions to obtain a score plot of the trend between group K and group $\mathrm{M}$. As can be seen from Fig. 4, on the 28th day of modeling, group $\mathrm{K}$ and group $\mathrm{M}$ could be distinguished clearly, revealing that the endogenous metabolic network of the body changed significantly after the rat model was established. To effectively find the endogenous markers that play a key role in clustering, the Ezinfo software was used to supervise the orthogonal partial least squares discriminant analysis (OPLS-DA) on the 28th day for the $\mathrm{K}$ and $\mathrm{M}$ rats. The results are shown in Fig. 5. S-plot and VIP-plot maps that directly reflect the key factors affecting the metabolic profile changes were obtained, as shown in Fig. 6 and 7. In the S-plot, the further the ion is from the origin, which contributes to the change in the metabolic profile, the more likely it is to be a potential biomarker. In the VIP scatter plot, the ion fragments are arranged in a V-shape, and the bottom ion (small VIP value) contributes less to the change in the metabolic contour trajectory and the top ion (large VIP value) contributes a large amount to the change in the metabolic contour trajectory. A scatter with a VIP value greater than 1 for a metabolic profile change was selected, a $T$ test value between the groups was selected, and a $P$ value of less than 0.05 was selected as the screening condition for a set of potential biomarkers. The RT and $m / z$ of important metabolite ions were
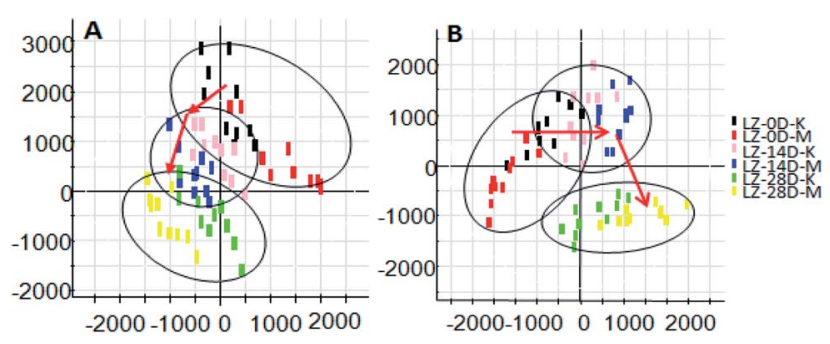

Fig. 3 Score plot of rat urine during $\mathrm{PCH}$ establishment of the hypertensive model (PCA analysis). A: positive ion mode and B: negative ion mode.
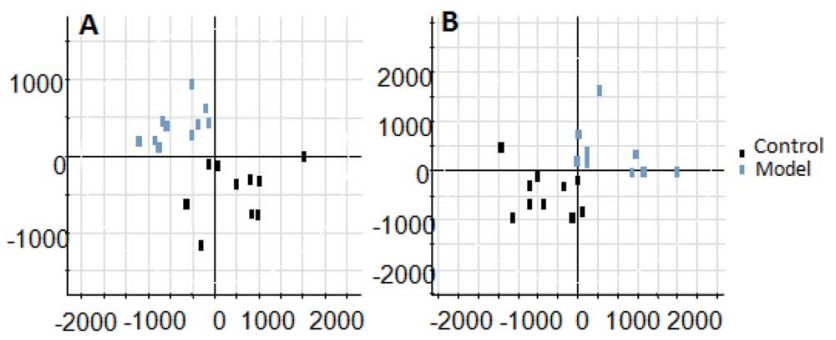

Fig. 4 Liver-fire type hypertensive model established on the 28th day, rat urine metabolism score plot (PCA analysis). A: positive ion mode and $\mathrm{B}$ : negative ion mode.

locked, and the $m / z$ was extracted from the BPI total ion flow chart (Fig. 8 and 9) to analyze the elemental composition, and the possible chemical formula was obtained. Based on the possible chemical formula and accurate mass and secondary mass spectrometry data, the HMDB and MetaboAnalyst websites were searched to match the mass spectrometry information and the attributes of the metabolites. The MassFragment module in the MassLynx software was used to determine the c hypertensionrelated metabolites. Thirty-two potential biomarkers were initially identified, as shown in Table S2. $\dagger$ The information on the secondary ion fragments of the 32 compounds is shown in Table S3. $\uparrow$ The changes in the content of each biomarker in groups $\mathrm{M}$ and $\mathrm{K}$ are shown in Fig. 10.

3.1.4 Metabolic pathway analysis of potential biomarkers in urine. The biomarkers of the identified 32 models of hepatic fire-fed hypertensive models were analyzed by MetPA, and 11 metabolic pathways (Table S4, $\uparrow$ Fig. 11 and 12) were disturbed, including phenylalanine, tyrosine and tryptophan biosynthesis, tyrosine metabolism, phenylalanine metabolism, tryptophan metabolism, ubiquinone and other terpenoid-quinone biosynthesis, pentose and glucuronate interconversions, starch and sucrose metabolism, vitamin B6 metabolism, histidine metabolism, lysine degradation, and aminoacyl-tRNA biosynthesis. The results indicate that these endogenous metabolites have a strong perturbation throughout the metabolic trajectory and are closely related to the liver-fire type hypertensive model.

\subsection{Pharmacological effect of Luozhen capsule}

3.2.1 Determination of blood pressure. Compared with group $\mathrm{K}$, the blood pressure of the other five groups increased significantly on the 28th day $(P<0.01)$, and compared with
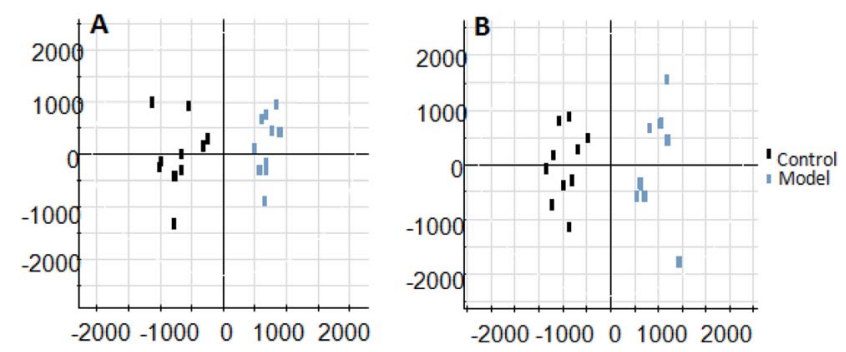

Fig. 5 Liver-fire type hypertensive model established on the 28th day of rat urine metabolism score plot (OPLS-DA analysis). A: positive ion mode and $B$ : negative ion mode. 

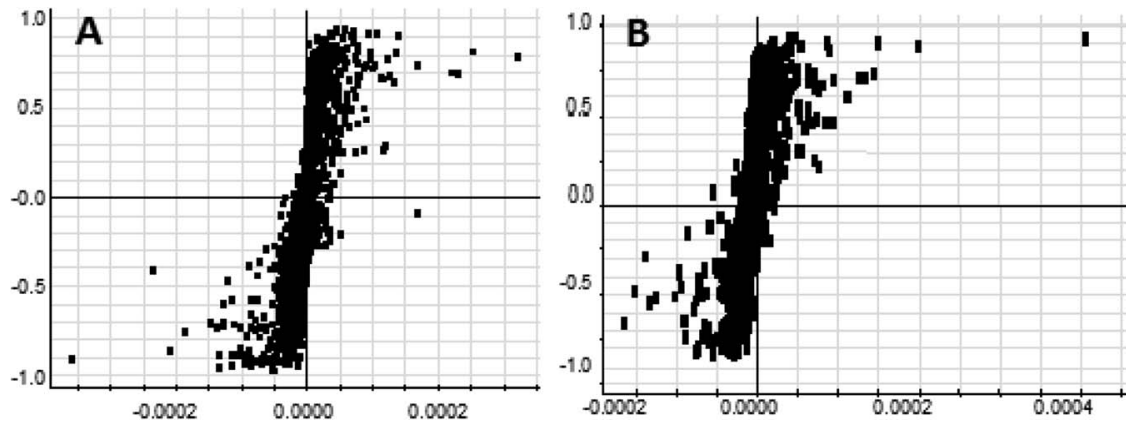

Fig. 6 S-plot map of urine metabolism profile of the rats in the positive and negative ion mode on the 28th day of the liver-fire type hypertensive rat model (OPLS-DA analysis). A: positive ion mode and B: negative ion mode.

group $\mathrm{M}$, the blood pressure of groups $\mathrm{Y}$ and $\mathrm{Z}$ decreased $(P<$ $0.05)$, while that of group $\mathrm{H}$ decreased significantly $(P<0.01)$. This shows that group $\mathrm{H}$ effectively inhibited the development of hypertension in the hyperactivity of the liver-fire model rats; group $\mathrm{Y}$ and group $\mathrm{Z}$ also have a certain effect, but the effect is not obvious; and group L basically does not have a therapeutic effect on the disease, the results are shown in Fig. 13.

3.2.2 Biochemical features. Compared with group $\mathrm{K}$, the serum levels of ANGII, NE and 5-HT in group M increased $(P<$ 0.05). Compared with group $M$, the serum levels of ANGII, NE and 5 -HT in group $\mathrm{H}$ were significantly reduced $(P<0.01)$. The results showed that group $\mathrm{H}$ group significantly improved the hypertension of the liver-fire model rats, and groups $\mathrm{H}, \mathrm{L}$ and $\mathrm{Y}$ had almost no therapeutic effect, and the results are shown in Fig. 14.

\subsection{Metabolomics study}

3.3.1 Changes in urinary metabolomic profile after oral administration of Luozhen capsule. Visual analysis showed that the urine samples of the different groups were slightly different, as shown in Fig. 15 and 16. Specifically, 6 groups of samples were analyzed for intra-group clustering and inter-group separation, and groups $\mathrm{K}$ group and $\mathbf{M}$ group were clearly distinguished, indicating that after modeling, the endogenous metabolites in the urine of the rats were significantly disturbed. Also, the $\mathrm{H}$ group clustered and the vector position approached the $\mathrm{K}$ group, indicating that the high dose of oral Luozhen capsule improved the liver-fire hypertensive model rats, as shown in Fig. 17.

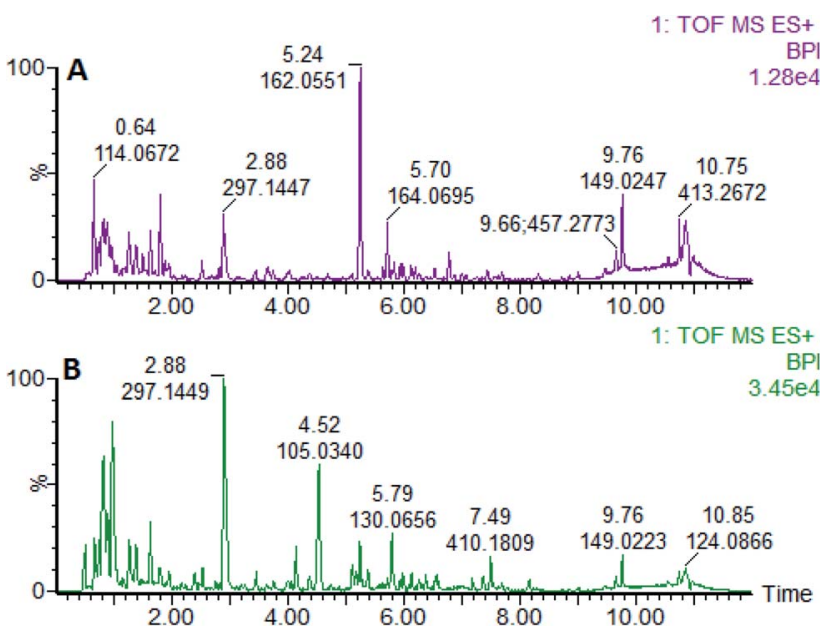

Fig. 8 Liver BPI of the rats in positive ion mode on the 28th day established by the model of high blood pressure. A: model group and B: control group.

3.3.2 Potential biomarkers in urine after oral administration of Luozhen capsules. The potential biomarkers in the model group were used as a reference, and the changes in the biomarkers with different doses of Luozhen capsules were analyzed. The results showed that oral Luozhen capsules could make the potential biomarker content of the rat models match that of group $\mathrm{K}$. The 32 potential biomarkers of urine identified
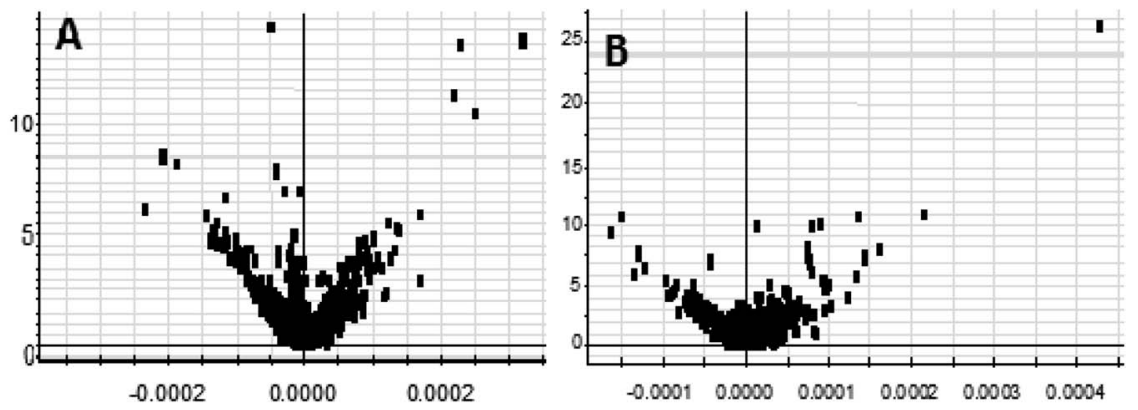

Fig. 7 Urine metabolism profile of the rats in the positive and negative ion mode on the 28th day of the liver-fire type hypertensive rat model (OPLS-DA analysis). A: positive ion mode and B: negative ion mode. 

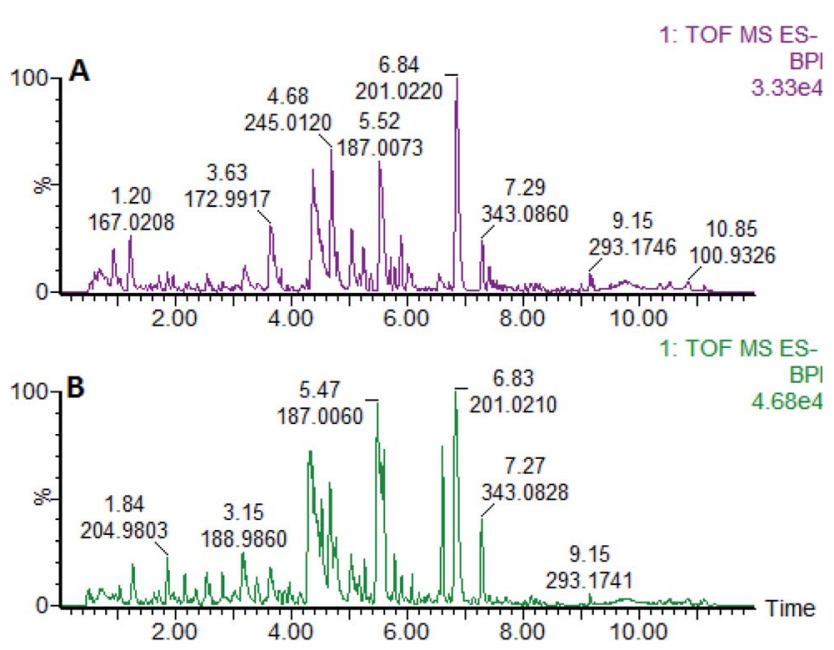

Fig. 9 Liver BPI of the rats in the negative ion mode established on the 28th day of the liver-fire hypertensive model. A: model group and B: control group.

in the second chapter were studied. After oral administration of Tianma Gouteng granules and Luozhen capsules, there was a trend of reversal. Among them, 12 of group Y disappeared and 18 of group $\mathrm{H}$ disappeared. In group Z, 10 of them disappeared and in group L, 9 of them disappeared. Also, 8 markers were found that could be adjusted in three doses of Luozhen capsules, 1-methylhistidine, 2-hydroxyphenethylamine, phenylacetaldehyde, pipecolic acid, dopaquinone, L-tyrosine, $\mathrm{N}$ acetylarylamine, and 4-hydroxyphenylpyruvic acid, as shown in Fig. 18.

\section{Discussion}

Metabolomics originated in the 1990s, and is now a mainstream subject of biological systems for the analysis of metabolites. ${ }^{18}$ It

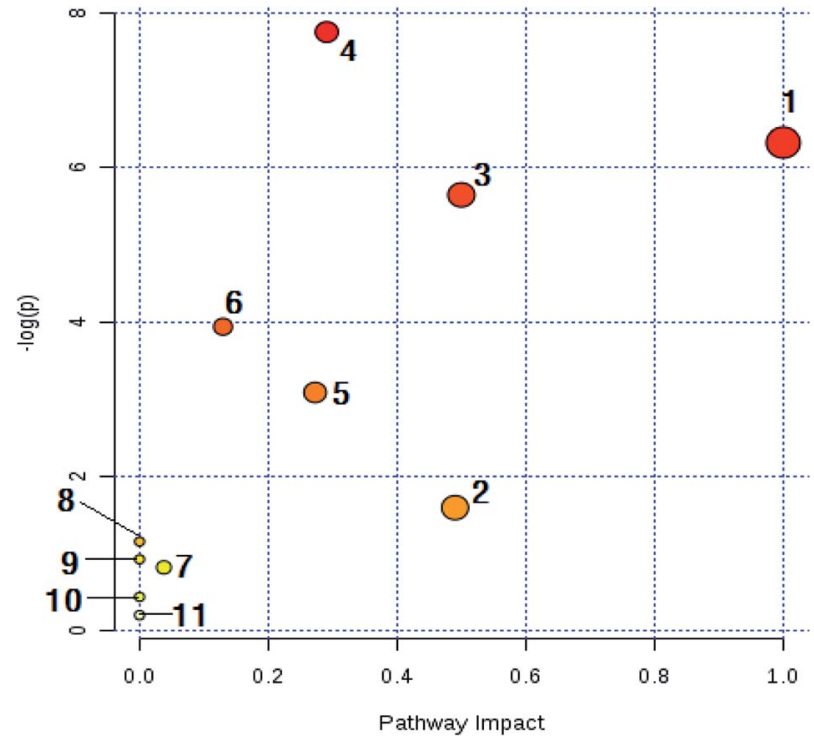

Fig. 11 Main metabolic pathways of potential biomarkers in liver-fire type hypertension. (1) Ubiquinone and other steroidal biosynthesis; (2) vitamin B6 metabolism; (3) phenylalanine, tyrosine and tryptophan biosynthesis; (4) tyrosine metabolism; (5) pentose and glucuronic acid conversion; (6) phenylalanine metabolism; (7) starch and sucrose metabolism; (8) histidine metabolism; (9) lysine degradation; (10) tryptophan metabolism; and (11) aminoacyl-tRNA biosynthesis.

is mainly used to study the types and quantitative changes of small molecule compounds of substrates or products of various metabolic pathways. ${ }^{19}$ Metabolomics can skip the biological regulation and directly analyze metabolites, and can be used to study the changed genes and proteins without the need for whole-genome detection and database establishment, fully exerting its versatility in the metabolic process. ${ }^{20}$ To provide further information on disease treatment targets, metabolic
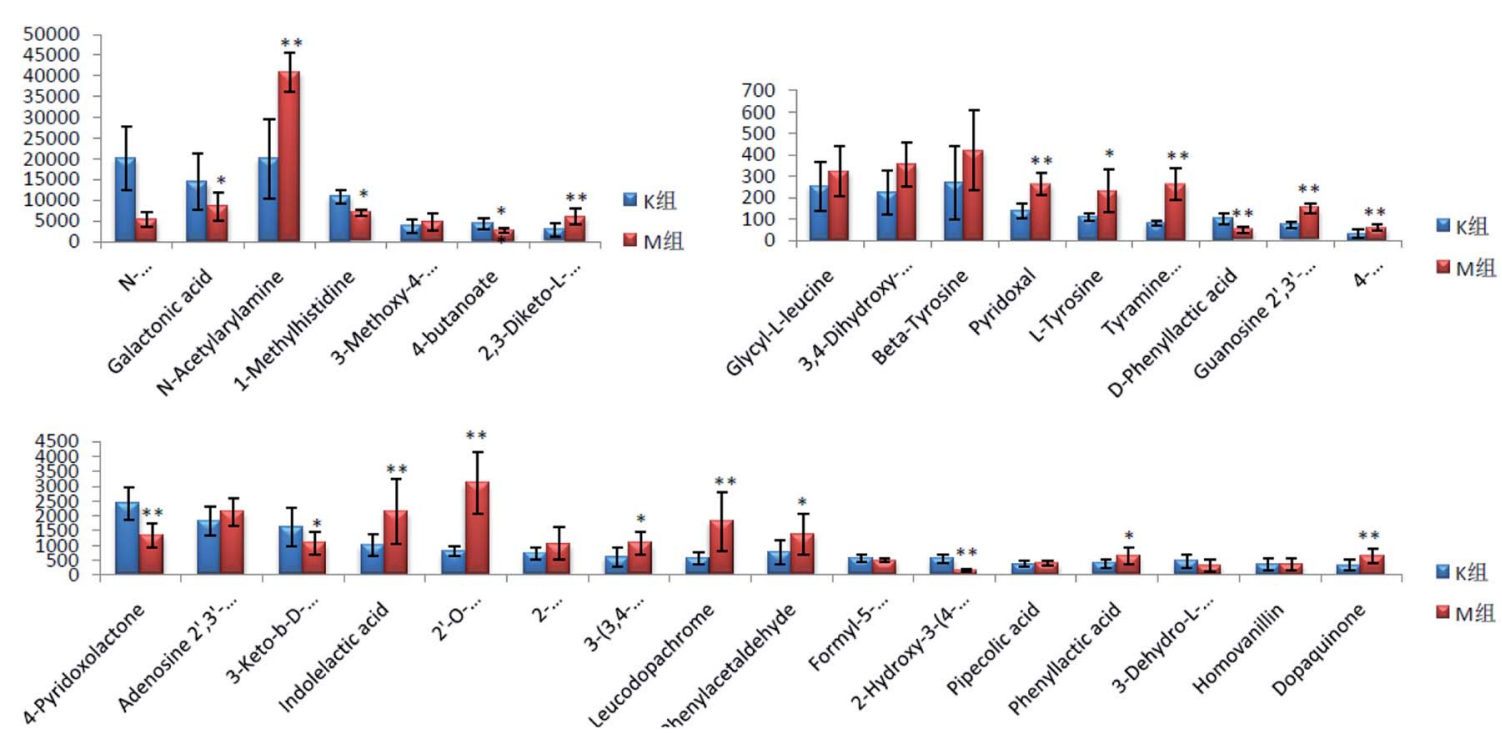

Fig. 10 Changes in the urine content of potential biomarkers for liver-fire type hypertension (compared with the control group, $* P<0.05, * * P<$ 0.01). 


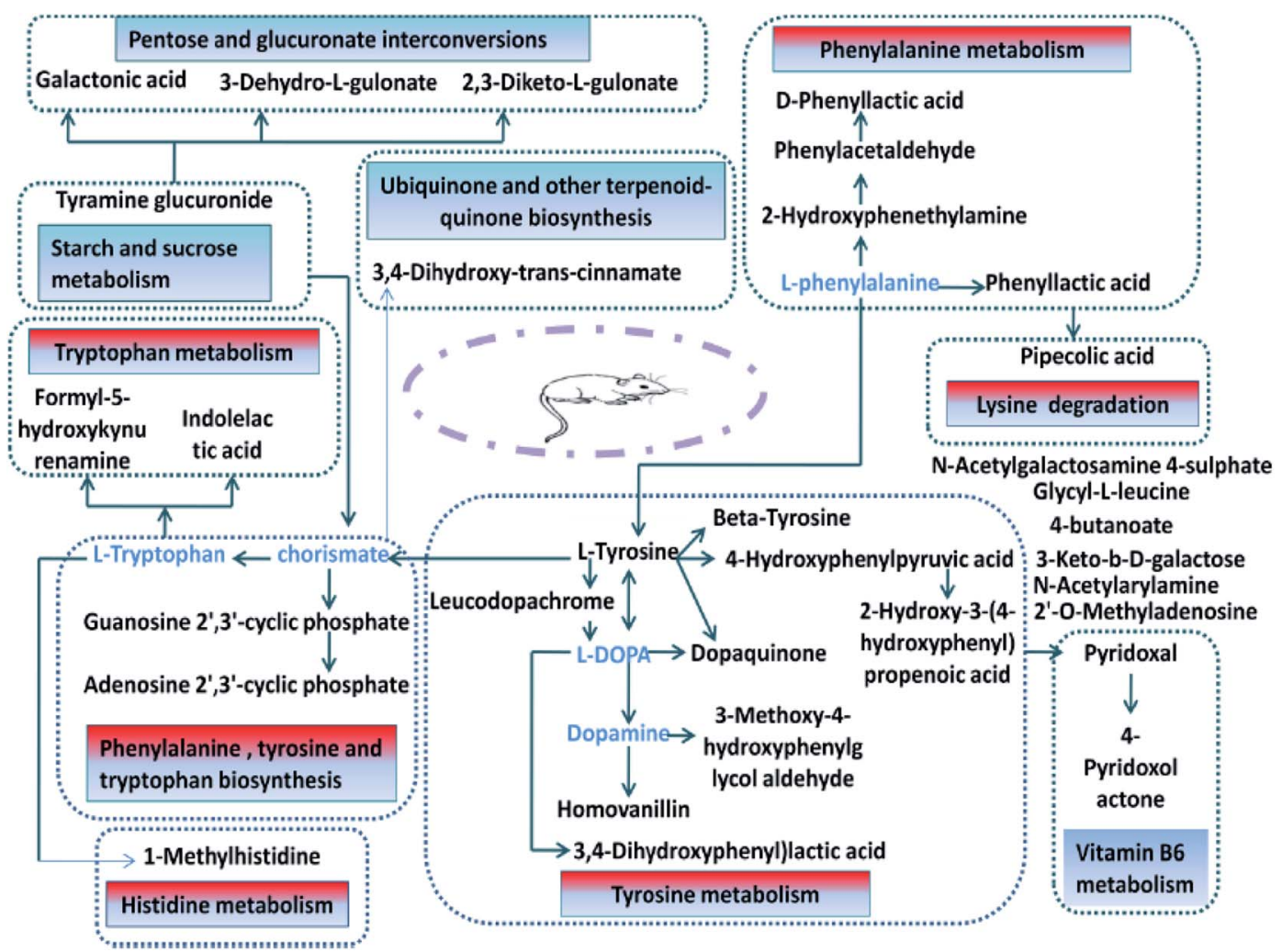

Fig. 12 Network diagram of the potential biomarkers associated with the liver-fire hypertensive model rats based on the KEGG network.

levels and changes in biological phenotypes are directly correlated, and then biomarkers are screened to reveal the metabolic mechanisms of the disease. ${ }^{21,22}$ Nowadays, metabolomics is great significance in the screening of models, the exploration of mechanisms and syndromes, and the clarification of related disease relationships. ${ }^{23}$ To improve the metabolomics platform, it should be further developed in the direction of highthroughput, high-sensitivity, high-resolution and multidimensional technology. ${ }^{24}$ Wang et al. studied the protective effect of Yinchenhao decoction on liver injury in rats. It was found that the metabolome of rats with liver injury had significant changes compared with the normal group. After the intervention of Yinchenhao decoction, the metabolic group of liver injury gradually returned to a level close to normal, showing a trend of callback, giving a new explanation for the prevention and treatment of liver damage by classical prescriptions from the perspective of drug metabolomics. ${ }^{25} \mathrm{Yu}$ et al. used urinary metabolomics based on ultra-high performance liquid chromatography coupled with quadruple time-offlight mass spectrometry for delineating the metabolic changes and potential early biomarkers in APPswe/PS1dE9 (APP/PS1) transgenic mice. It was shown that 24 differential metabolites

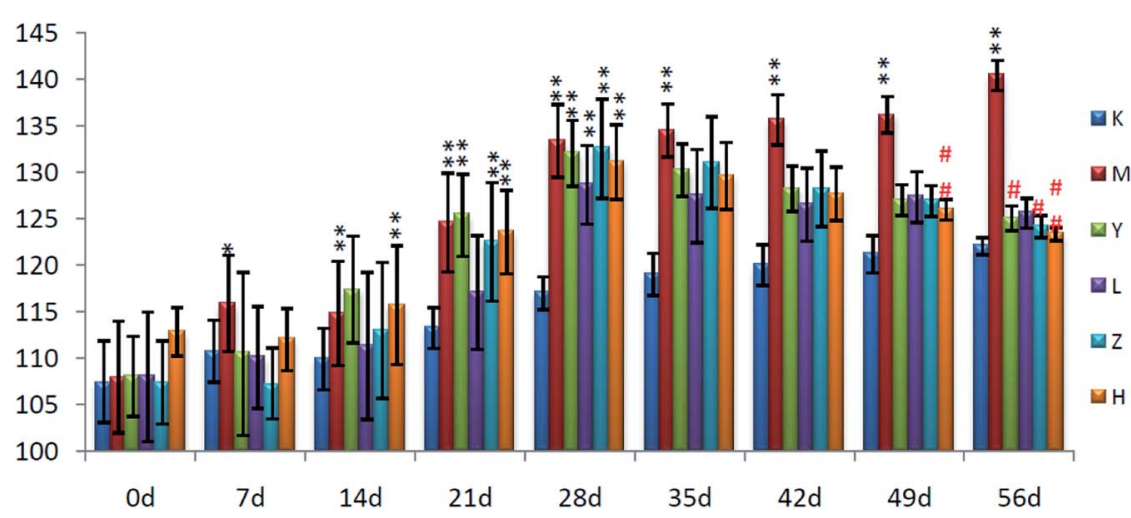

Fig. 13 Effect of Luozhen capsules on the blood pressure of rats with liver-fire type hypertensive model $(* P<0.05, * * P<0.01$, compared with group K; ${ }^{\#} P<0.05,{ }^{\# \#} P<0.01$, compared with the Model); K: control group; M: model group; Y: positive group; L: LZC low-dose group; Z: LZC medium-dose group; and H: LZC high-dose group. 

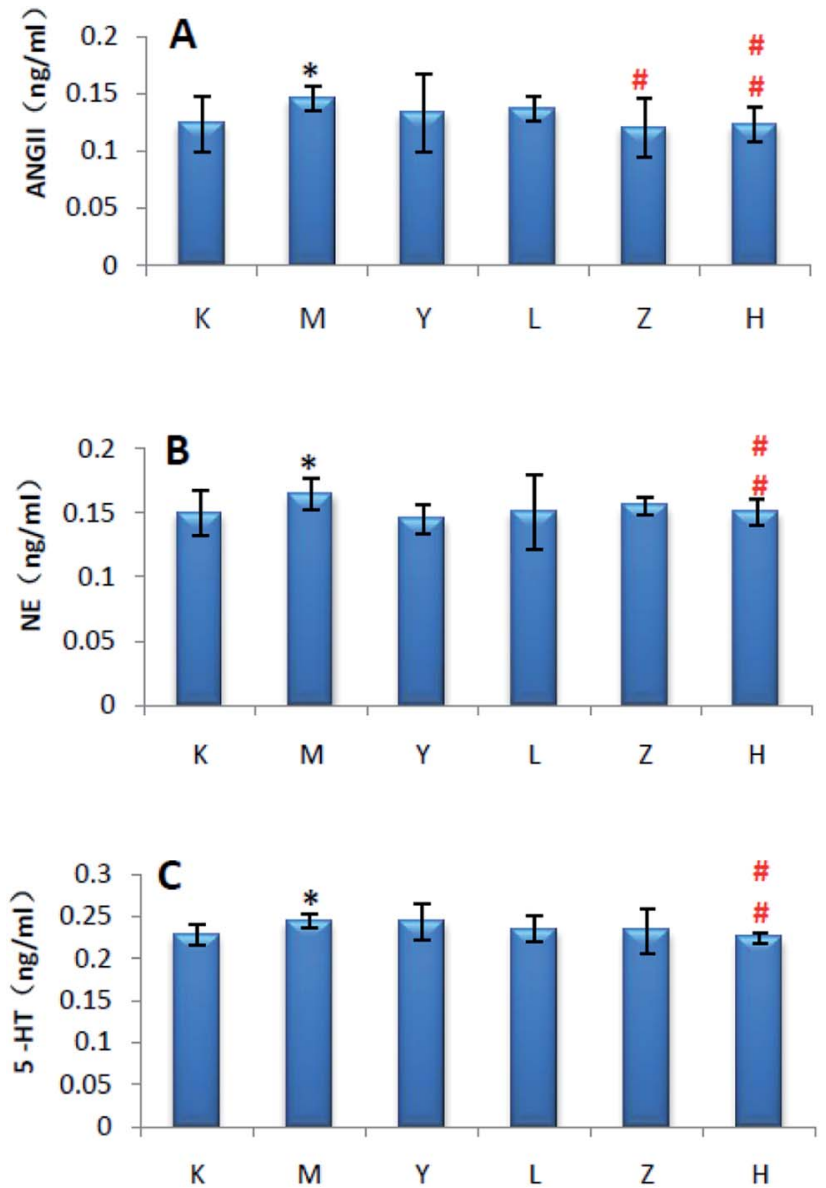

Fig. 14 Effect of Luozhen capsules on the clinical biochemical parameters of rats with liver-fire type hypertensive model $(* P<0.05$, ${ }^{* *} P<0.01$ compared with the control; ${ }^{\#} P<0.05,{ }^{\# \#} P<0.01$, compared with the Model). K: control group; M: model group; $Y$ : positive control group; L: LZC low dose group; Z: LZC medium dose group; and H: LZC high dose group. A: ANGIl; B: NE; and C: 5-HT.

were identified in the transgenic mice, which can serve as potential biomarkers for Alzheimer's disease, involving the two most relevant metabolic pathways for NO production pathway and metabolism of monoaminergic neurotransmitters. ${ }^{26}$ Through the comprehensive performance of all the metabolites of graduate students, the model was evaluated, and the overall function and mechanism of Chinese medicine and its combination could be interpreted. As an effective way to evaluate the effect of traditional Chinese medicine, metabolomics can comprehensively reveal a series of biochemical changes in biological systems during the treatment of Chinese medicine and complex diseases. Thus, it is possible to evaluate the pharmacodynamic effects and the material basis and mechanism of action for discovering the effects of drugs.

Traditional Chinese medicine uses a unique holistic, dialectical, and macroscopic scientific theory system to diagnose diseases, but it lacks the modern scientific interpretation of its mechanism. Metabolomics studies the endogenous substances produced by the external environment. The overall response, which encompasses the entire body response, is a perfect match
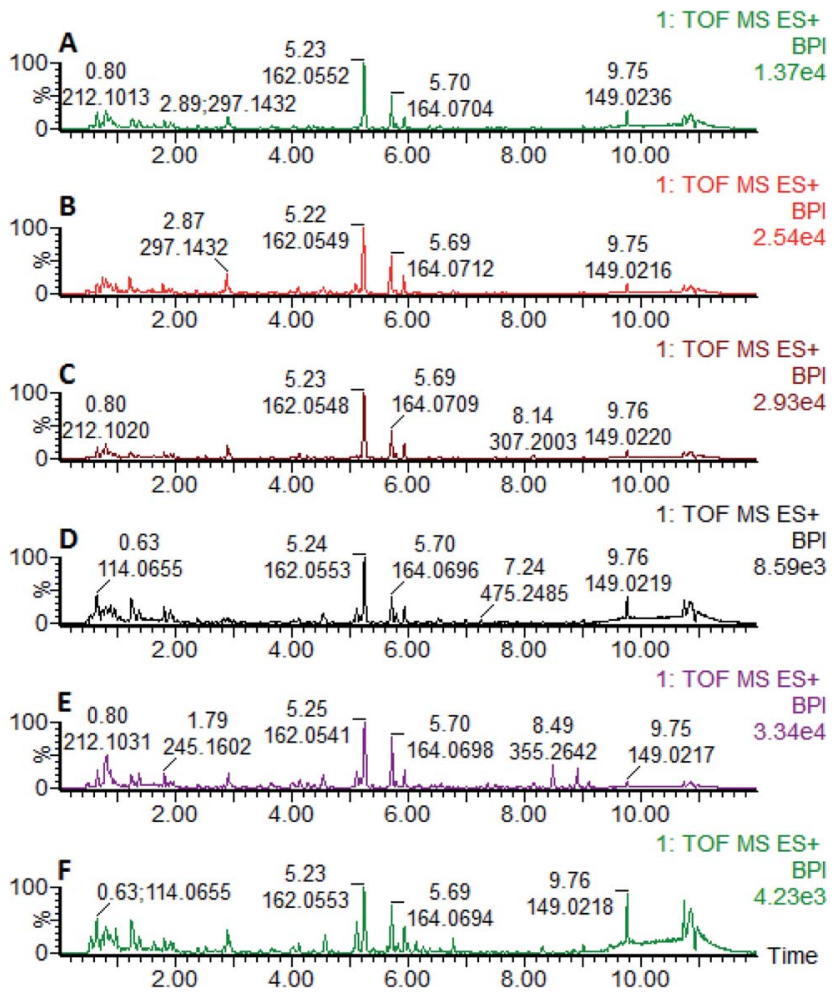

Fig. 15 In positive ion mode, after oral administration of Luozhen capsules, urine BPI map of the 56th day with hypertensive model rat of excessive liver-fire syndrome. A: control group; B: model group; C: positive control group; D: low dose group; E: medium dose group; and F: high dose group.

with traditional Chinese medicine. ${ }^{27}$ The role of traditional Chinese medicine is to pay attention to the overall integrity, multi-component, multi-target, level and network complexity. Metabolomics comprehensively grasps the characteristics of traditional Chinese medicine, and determines the concentration and composition of related metabolites caused by traditional Chinese medicine through the latest technical analysis methods. The above changes, in turn, analyze the changes in related protein and gene expression, and clarify the mechanism of action of Chinese medicine at a higher level. ${ }^{28} \mathrm{Li} \mathrm{F}$. et al. ${ }^{29}$ used UPLC-MS technology to study the chemical constituents and in vivo ingredients of Epimedium. The results showed that Asarum C. and icariin are the main medicinal ingredients in Epimedium. Gu Y. et al. ${ }^{30}$ used berberine in the metabolomics analysis of type- 1 diabetes, and concluded that berberine can significantly down-regulate the level of free fatty acids.

In Luozhen capsules, the main component of action is the extract of Apocynum venetum leaves, which reduces blood and urine nitrogen levels, promotes NO release ${ }^{31}$ and affects $\mathrm{K}^{+}$and $\mathrm{Na}^{+}$channels. ${ }^{32}$ Thus it exerts its antihypertensive effect by dilating the blood vessels in the endothelium in a concentration-dependent manner. ${ }^{33}$ Astragaloside and gammaaminobutyric acid in the Astragalus component may act to reduce blood pressure by expanding blood vessels. ${ }^{34-36}$ Salvia miltiorrhiza contains the main antihypertensive component of danshensu, which enhances the expression and production of 

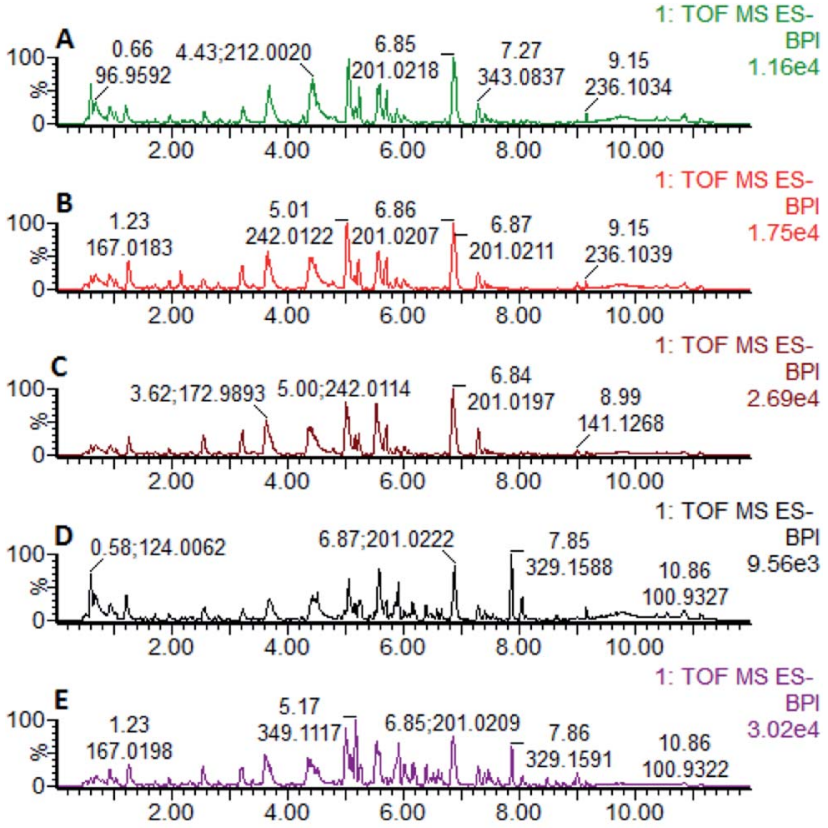

1: TOF MS ES-

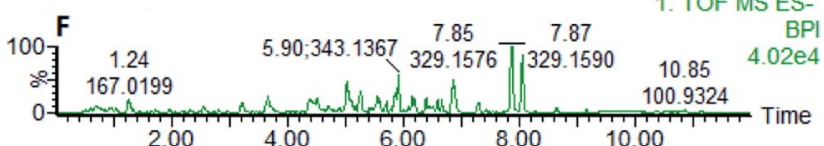

Fig. 16 In negative ion mode, after oral administration of Luozhen capsules, urine BPI map of the 56th day of liver-fire hypertensive rats. A: control group; B: model group; $C$ : positive control group; D: low dose group; E: medium dose group; and F: high dose group.

cyclooxygenase-2 and prostacyclin in endothelial cells, ${ }^{37}$ opening a part of $\mathrm{K}^{+}$channels, and thus inhibiting the influx of $\mathrm{Ca}^{2+}$ in vascular smooth muscle cells. This allows vasodilation to improve hypertension. ${ }^{38}$ Ligustilide, a compound of benzoquinone, can relieve myocardial hypertrophy caused by AngII in a dose-dependent manner, reduce the expression of Bcl-2, p53 and Bax, and relieve blood pressure by relaxing blood vessels. ${ }^{39}$ The main chemical component of paeony, paeoniflorin, is defined by controlling the activity of the liver and regulating the concentration of endothelin (reduced) ${ }^{\mathbf{4 0}}$ and nitric oxide (elevation $)^{\mathbf{4 1}}$ in serum. In liver and blood pressure reduction [340], curcumin in turmeric inhibits the release of proinflammatory factors by regulating nuclear factor- $\kappa \mathrm{B}$, and finally plays an anti-inflammatory and hepatoprotective role. ${ }^{\mathbf{4 2}}$ The orange cassia in Cassia is one of the main active ingredients in cassia seed, which can cause vasodilation in a dosedependent manner to achieve an antihypertensive therapeutic effect. The volatile oil in the pine is the main component for the reduction of blood pressure. On the one hand, it can induce endothelium-dependent relaxation and relaxation by regulating acetylcholine (Ach), thereby promoting the release of nitric oxide (NO) from endothelial cells; on the other hand, it also can directly increase the level of $\mathrm{Ca}^{2+}$ in cells to promote the release of NO. ${ }^{43,44}$

In this study, aconite, dried ginger and cinnamon extracts were combined with L-NAME to establish a liver-fire type hypertensive rat model. The model was evaluated by blood pressure and clinical biochemical indicators. The blood pressure results showed that the blood pressure of the model rats increased significantly beyond the normal range. The results of clinical biochemical indicators showed that the serum levels of ANGII, NE and 5-HT in the model group increased substantially. The above results prove that this model was successfully established. The UPLC-MS technique was used to analyze the difference in urine metabolism profiles between the rats with the hypertensive model of hyperactivity of liver-fire and the control group by means of metabolomics. It can be seen that the model group and the control group were clearly clustered, according to metabolomics. The metabolic network and pathway have previously been established for the search for potential drug targets in disease treatment. ${ }^{45-56}$ Disease-related biomarkers were identified and identified by searching HMDB, KEGG, MetPA and other databases, including galactonic acid, 3-keto- $\beta$-D-galactose, $N$-acetylgalactosamine 4 sulphate, 1-methylhistidine, 2-hydroxyphenethylamine, phenylacetaldehyde, 2,3-diketo-L-gulonate, 2'-O-methyladenosine, 4-butanoate, tyramine glucuronide, adenosine $2^{\prime}, 3^{\prime}$-cyclic phosphate, 4-pyridoxolactone, guanosine $2^{\prime}, 3^{\prime}$-cyclic phosphate, pipecolic acid, glycyl-s-leucine, pyridoxal, formyl-5hydroxykynurenamine, 3-(3,4-dihydroxyphenyl) lactic acid,
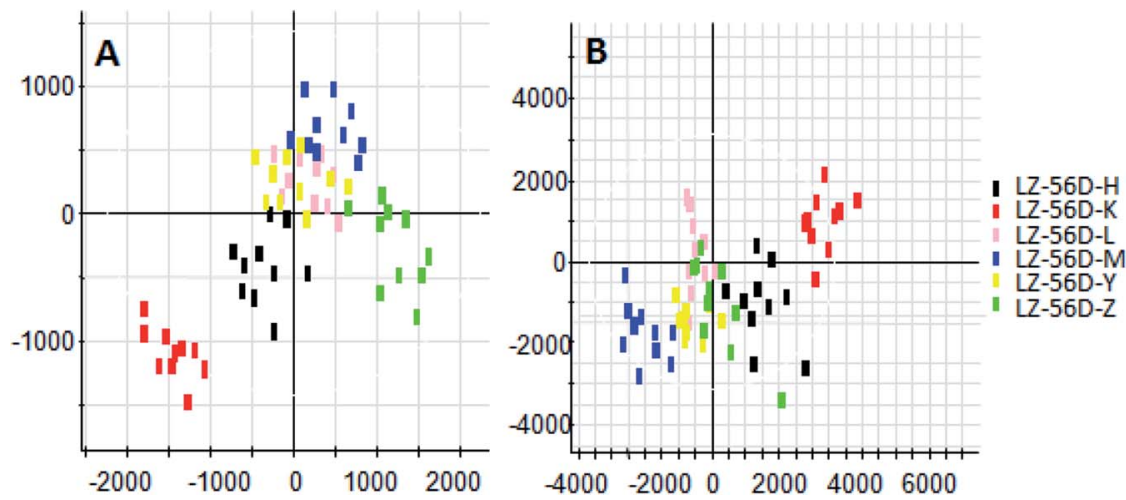

Fig. 17 Score plot of urine metabolism profile on day 56 of hypertensive rats after oral administration of Luozhen capsules (PCA analysis). A: positive ion mode and B: negative ion mode. 

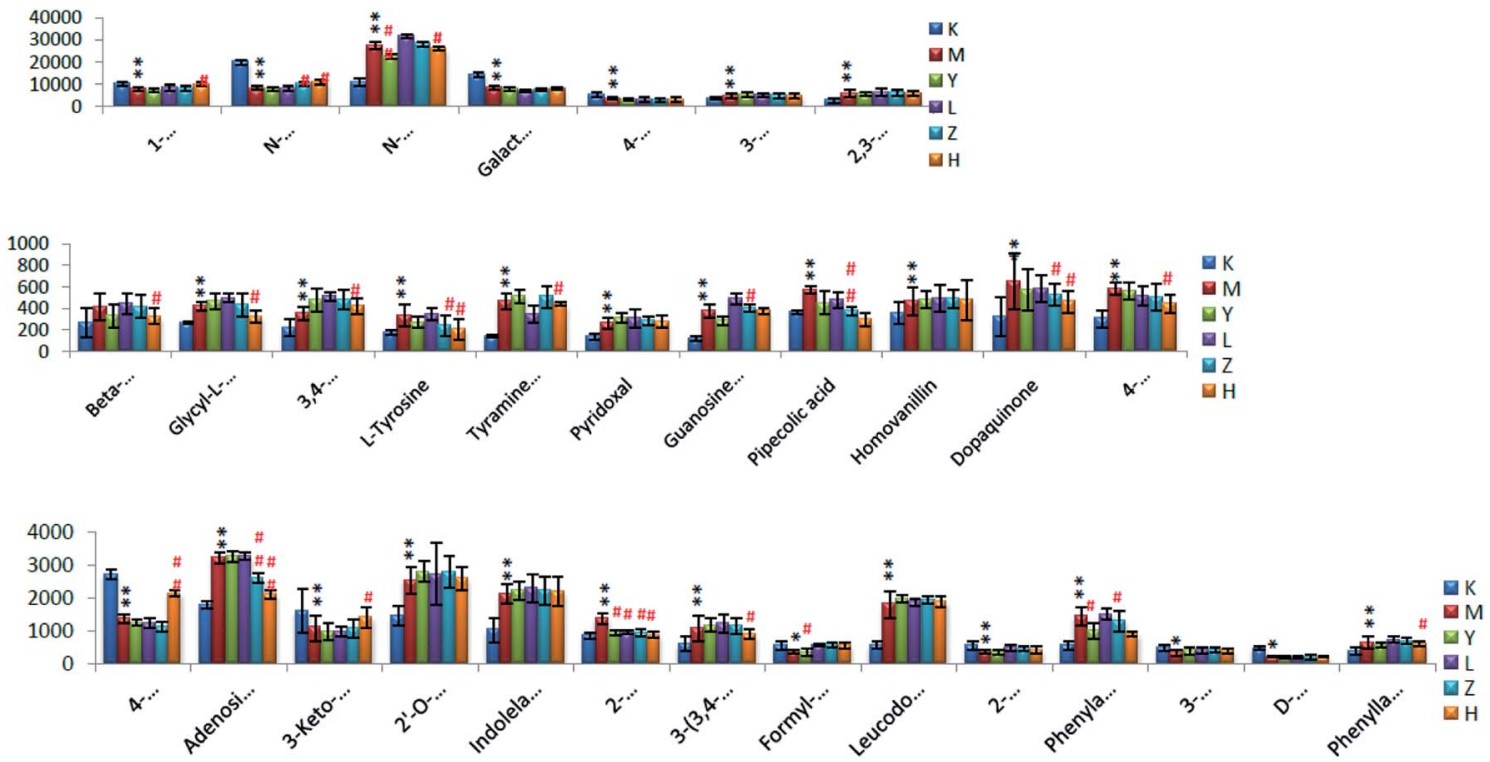

Fig. 18 Changes in the relative urine content of biomarkers in liver-burning hypertensive patients after oral administration of Luozhen capsules $\left({ }^{*} P<0.05, * * P<0.01\right.$ compared with blanks; ${ }^{\#} P<0.05,{ }^{\# \#} P<0.01$, compared with the model) K: control group; M: model group; Y: positive group; L: LZC low dose group; Z: LZC medium dose group; and H: LZC high dose group.

dopaquinone, 3-methoxy-4-hydroxyphenylglycol aldehyde, betatyrosine, L-tyrosine, 3,4-dihydroxy-trans-cinnamate, homovanillin, D-phenyllactic acid, $N$-acetylarylamine, 4-hydroxyphenylpyruvic acid, leucodopachrome, 2-hydroxy-3-(4hydroxyphenyl) propenoic acid, and phenyllactic acid and 32 potential endogenous biomarkers such as indolelactic acid, and 3-dehydro-L-gulonate. Bioinformatics methods were used to analyze and determine the major metabolic pathways involved, including phenylalanine, tyrosine and tryptophan biosynthesis, tyrosine metabolism, phenylalanine metabolism, tryptophan metabolism, ubiquinone and other terpenoid-quinone biosynthesis, pentose and glucuronate interconversions, starch and sucrose metabolism, vitamin B6 metabolism, histidine metabolism, lysine degradation, and aminoacyl-tRNA biosynthesis.

Furthermore, using the liver-fire hypertensive model and Luozhen capsules as experimental subjects, the endogenous biomarkers of the model rats were analyzed. After oral administration of different doses of Luozhen capsules in the model rats, it can be seen from the physiological and biochemical indexes of the model rats that large doses significantly improved hypertension in excessive liver-fire syndrome. It was found that oral Luozhen capsules can affect the potential biomarkers of the model, which can be adjusted back to that of the blank group. In the urine samples, eight biomarkers were initially identified to be called back in high, medium and low doses of Luozhen capsules, including 1-methylhistidine, 2hydroxyphenethylamine, phenylacetaldehyde, pipecolic acid, dopaquinone, L-tyrosine, $\mathrm{N}$-acetylarylamine, and 4-hydroxyphenylpyruvic acid. By tracking the biomarkers in the urine samples, it is highly speculated that the main metabolic pathway regulated by Luozhen capsules is amino acid metabolism. The mechanism of hypertension is mainly an important regulation of vasomotor nerves. Sensory nerves release a variety of vasoactive neuropeptides, of which calcitonin gene-related peptide (CGRP) is an important neurotransmitter of capsaicinsensitive sensory nerves and is widely distributed in the central, peripheral and other systems. It consists of 37 amino acids, and is the strongest vasodilator known to have the effect of lowering blood pressure, lowering peripheral resistance, renal artery relaxation, and a significant increase in renal blood flow. 4-Hydroxyphenylpyruvic acid is a metabolic intermediate of phenylalanine. The side chain of phenylalanine is hydroxylated to tyrosine by phenylalanine hydroxylase. Tyrosine is in turn converted to 4-hydroxyphenylpyruvate by tyrosine transaminase. Tyrosine is an essential amino acid that can relieve stress and has a good therapeutic effect on depression, anxiety, and allergic headaches. Related literature shows that tyrosine has identified biomarkers for essential hypertension. More tyrosine and methionine are needed during sympathetic activation, which may be an important mechanism for $\mathrm{EH}$. Next, our task is to continue the study of blood metabolomics, and the metabolic data of urine and blood together to determine the endogenous biomarkers of the liver-fire hypertensive syndrome and the affected metabolic pathways.

\section{Conclusion}

Hypertension with hyperactivity of liver-fire is a life-long illness that tends to occur in younger people. Since this disease needs life-long treatment, it causes great inconvenience to life and work. Cardiovascular diseases caused by hyperactivity of liverfire result in the death of humans. The etiology of the disease is complex, meanwhile its occurrence and development are affected by many factors, which lead to vasoconstriction, injury, remodeling and continuous increase in blood pressure. Compared with the control group, 32 variables were found in 
the model rat, which could be used as potential of the disease and involved 11 metabolic pathways. After taking Luozhen capsules orally, 8 of the 32 biomarkers changed, leading to the preliminary speculation of amino acid metabolism. The next step will focus on the above biomarkers and metabolic pathways for targeted research in order to clarify the mechanism with hypertension of hyperactivity liver-fire type and relate it to essential hypertension to provide a theoretical basis. This lays a foundation for discovering the pharmacodynamic substance basis of Luozhen capsules in the treatment of this sickness, and hopes to make achievements in the field of new drug research and development.

\section{Conflicts of interest}

There are no conflicts to declare.

\section{Acknowledgements}

This work was supported by grants from the Key Program of Natural Science Foundation of State (Grant No. 81830110, 8181101160, 81973745, 81903818, 81903847, 81430093, 81673586, 81703685, 81302905, 81503386, 81373930), National Key Research and Development Program of China (2018YFC1706103), National Key Subject of Drug Innovation (Grant No. 2015ZX09101043-005, 2015ZX09101043-011), TCM State Administration Subject of Public Welfare (Grant No. 2015468004), Major Projects of Application Technology Research and Development Plan in Heilongjiang Province (GX16C003), Natural Science Foundation of Heilongjiang Province (YQ2019H030, LH2019H056, H2016056), Chinese Postdoctoral Science Foundation (2017M621319b), University Nursing Program for Young Scholars with Creative Talents in Heilongjiang Province (UNPYSCT-2015118, UNPYSCT-2016213, UNPYSCT-2016212), Young Talent Lift Engineering Project of China Association of Traditional Chinese Medicine (QNRC2B06), Foundation of Heilongjiang University of Chinese Medicine (2018jc01, 2018bs02, 201809).

\section{References}

1 C. Farsang, L. Naditchbrule, A. Avogaro, et al., Where are we with the management of hypertension? From science to clinical practice, J. Clin. Hypertens., 2010, 11(2), 66-73.

2 H. Nishijima, R. Haga, C. Suzuki, et al., Asymmetric Posterior Reversible Encephalopathy Syndrome due to Hypertensive Encephalopathy, Intern. Med., 2015, 54(8), 993-994.

3 Q. Li, L. Chen, D. Chen, et al., Influence of microRNA-related polymorphisms on clinical outcomes in coronary artery disease, Am. J. Transl. Res., 2015, 7(2), 393-400.

4 X. Liu, L. You, R. Zhou, et al., Significant association between functional microRNA polymorphisms and coronary heart disease susceptibility: a comprehensive meta-analysis involving 16484 subjects, OncoTargets, 2017, 8(4), 5692.

5 M. H. Forouzanfar, P. Liu, G. A. Roth, et al., Global Burden of Hypertension and Systolic Blood Pressure of at Least 110 to
$115 \mathrm{~mm} \mathrm{Hg}$, 1990-2015, JAMA, J. Am. Med. Assoc., 2017, 317(2), 165-182.

6 C. Leimena and H. Qiu, Non-Coding RNA in the Pathogenesis, Progression and Treatment of Hypertension, Int. J. Mol. Sci., 2018, 19(4), 927.

7 G. R. Drummond, A. Vinh, T. J. Guzik and G. Christopher, Immune mechanisms of hypertension, Nat. Rev. Immunol., 2019, 19(8), 517-532.

8 Y. Xia and R. E. Kellems, Angiotensin Receptor Agonistic Autoantibodies and Hypertension: Preeclampsia and Beyond, Circ. Res., 2013, 113(1), 78-87.

9 F. C. Luft, Activating autoantibodies and cardiovascular disease, Physiology, 2013, 28(4), 254.

10 A. K. Johnson and B. Xue, Central nervous system neuroplasticity and the sensitization of hypertension, Nat. Rev. Nephrol., 2018.

11 S. M. Al-Khatib, W. G. Stevenson, M. J. Ackerman, W. J. Bryant, D. J. Callans, A. B. Curtis, B. J. Deal, T. Dickfeld, M. E. Field, G. C. Fonarow, A. M. Gillis, C. B. Granger, S. C. Hammill, M. A. Hlatky, J. A. Joglar, G. N. Kay, D. D. Matlock, R. J. Myerburg and R. L. Page, AHA/ACC/HRS Guideline for Management of Patients With Ventricular Arrhythmias and the Prevention of Sudden Cardiac Death: Executive Summary: A Report of the American College of Cardiology/American Heart Association Task Force on Clinical Practice Guidelines and the Heart Rhythm Society, J. Am. Coll. Cardiol., 2018, 72(14), 1677-1749.

12 L. Jie-Wei, L. Cong-Huai, Z. Yao-Bin, et al., Association of Tongue Bacterial Flora and Subtypes of Liver-Fire Hyperactivity Syndrome in Hypertensive Patients, J. Evidence-Based Complementary Altern. Med., 2018, 2018, 110.

13 L. Jie-Wei, L. Cong-Huai, Z. Yao-Bin, et al., Association of Tongue Bacterial Flora and Subtypes of Liver-Fire Hyperactivity Syndrome in Hypertensive Patients, J. Evidence-Based Complementary Altern. Med., 2018, 2018, 110.

14 X. Y. Zheng, Guidelines for clinical research on Chinese new herbal medicines, Medical Science and Technology Publishing House of China, Beijing, 2002, p. 163.

15 M. Humbert, N. W. Morrell, S. L. Archer, et al., Cellular and molecular pathobiology of pulmnary arterial hypertesion, $J$. Am. Coll. Cardiol., 2004, 43(12s1), s13-s24.

16 W. Xiu-Hong, Z. Chuang, Z. Ai-Hua, et al., High-throughput metabolomics used to identify potential therapeutic targets of Guizhi Fuling Wan against endometriosis of cold coagulation and blood stasis, RSC Adv., 2018, 8(34), 1923819250.

17 Y. Nan, X. Zhou, Q. Liu, et al., Serum metabolomics strategy for understanding pharmacological effects of ShenQi pill acting on kidney yang deficiency syndrome, Journal of Chromatography B Analytical Technologies in the Biomedical \& Life Sciences, 2016, 1026, 217-226.

18 A. C. Dona, K. Michael, S. Flora, et al., A guide to the identification of metabolites in NMR-based metabonomics/ 
metabolomics experiments:, Comput. Struct. Biotechnol. J., 2016, 14, 135-153.

19 J. K. Nicholson, J. Connelly, J. C. Lindon, et al., Metabonomics: a platform for studying drug toxicity and gene function, Nat. Rev. Drug Discovery, 2002, 1(2), 153-161.

20 A. Zhang, H. Sun and X. Wang, Power of metabolomics in biomarker discovery and mining mechanisms of obesity, Obes. Rev., 2013, 14(4), 344-349.

21 A. Zhang, H. Sun and X. Wang, Serum metabolomics as a novel diagnostic approach for disease: a systematic review, Anal. Bioanal. Chem., 2012, 404(4), 1239-1245.

$22 \mathrm{~W}$. Weckwerth, Unpredictability of metabolism-the key role of metabolomics science in combination with nextgeneration genome sequencing, Anal. Bioanal. Chem., 2011, 400(5), 1967-1978.

23 X. Wang, A. Zhang, Y. Han, et al., Urine metabolomics analysis for biomarker discovery and detection of jaundice syndrome in patients with liver disease, Mol. Cell. Proteomics, 2012, 11(8), 370-380.

24 A. Calabro, E. Gralka, C. Luchinat, et al., A metabolomic perspective on celiac disease, Autoimmune Dis., 2014, 8(6), $2-10$.

25 X. Wang, H. Lv, H. Sun, et al., Metabolic urinary profiling of alcohol hepatotoxicity and intervention effects of Yin Chen Hao Tang in rats using ultra-performance liquid chromatography/electrospray ionization quadruple time-offlight mass spectrometry, J. Pharm. Biomed. Anal., 2008, 48(4), 1161-1168.

$26 \mathrm{~J} . \mathrm{Yu}, \mathrm{L}$. Kong, A. Zhang, et al., High-throughput metabolomics for discovering potential metabolite biomarkers and metabolic mechanism from APPswe/ PS1dE9 transgenic model of Alzheimer's disease, $J$. Proteome Res., 2017, 16(9), 3219.

27 A. Zhang, H. Sun, G. Yan, et al., Metabomolics in diagnosis and biomarker discovery: Moving to the clinic, BioMed Res. Int., 2015, 2015, 354671.

28 A. Zhang, H. Sun, G. Yan, et al., Metabomolics in diagnosis and biomarker discovery of colorectal cancer, Cancer Lett., 2014, 345(1), 17-20.

29 F. Li, X. Lu, H. Liu, et al., A-pharmaco-metabonomic study on the Therapeutic hasis and metabolic effect of Epimedium brevicornum Maxim on hydrocortisoneinduced rat using UPLC-MS, Biomed. Chromatogr., 2007, 21(4), 397-402.

30 Y. Gu, Y. Zhang, X. Shi, et al., Effect of traditional Chinese medicine berberine on type 2 diabetes based on comprehensive metabonomics, Talanta, 2010, 81(3), 766.

31 C. Tagawa, T. Kagawa, Y. Nakazawa, et al., Studies on antihypertensive effect of Luobuma (Apocynum venetum L.) leaf extract (3), Yakugaku Zasshi, 2004, 124(11), 851-856.

32 C. Y. Kwan, W. B. Zhang, S. Nishibe, et al., A novel in vitro endothelium-dependent vascular relaxant effect of Apocynum venetum leaf extract, Clin. Exp. Pharmacol. Physiol., 2005, 32(9), 789-795.

33 L. Yeh, L. Wei, M. Dharmani, et al., Endothelium-Dependent Relaxation Effect of Apocynum venetum Leaf Extract via Src/
PI3K/Akt Signalling Pathway, Nutrients, 2015, 7(7), 52395253.

34 Q. Y. Yang, K. J. Chen, S. Lu, et al., Research progress on mechanism of action ofRadix Astragalusin the treatment of heart failure, Chin. J. Integr. Med., 2012, 18(3), 235-240.

35 D. Wang, G. Fan, Y. Wang, et al., Vascular reactivity screen of Chinese medicine danhong injection identifies Danshensu as a NO-independent but PGI2-mediated relaxation factor, J. Cardiovasc. Pharmacol., 2013, 62(5), 457.

36 N. Y. Li, X. L. Li, X. P. Zhai, et al., Effects of Mongolia Astragali Radix in protecting early cardiac and nephritic functions of patients of hypertension with metabolic syndrome, China J. Chin. Mater. Med., 2016, 41(21), 40514059.

37 F. F. Lam, J. H. Yeung, K. M. Chan, et al., Relaxant effects of danshen aqueous extract and its constituent danshensu on rat coronary artery are mediated by inhibition of calcium channels, Vasc. Pharmacol., 2007, 46(4), 271-277.

38 L. U. Qun, S. Luo and Y. Wen, Effect of ligustilide on Ang IIinduced hypertrophy in cardiomyocytes and the potential mechanisms, Exp. Ther. Med., 2014, 8(1), 169-174.

39 K. T. Kang, Endothelium-derived Relaxing Factors of Small Resistance Arteries in Hypertension, Toxicol. Res., 2014, 30(3), 141.

40 J. X. Zhang, J. R. Yang, G. X. Chen, et al., Sesamin ameliorates arterial dysfunction in spontaneously hypertensive rats via downregulation of $\mathrm{NADPH}$ oxidase subunits and upregulation of eNOS expression, Acta Pharmacol. Sin., 2013, 34(7), 912-920.

41 B. Li, Z. B. Yang, S. S. Lei, et al., Beneficial Effects of Paeoniflorin Enriched Extract on Blood Pressure Variability and Target Organ Damage in Spontaneously Hypertensive Rats, Evid.-Based Complementary Altern. Med., 2017, 2017, 5816960.

42 Y. Rivera-Espinoza and P. Muriel, Pharmacological actions of curcumin in liver diseases or damage.Liver, Int, 2009, 29(10), 1457-1466.

43 J. B. Zhang, M. L. Liu, C. Li, et al., Nardosinane-type sesquiterpenoids of Nardostachys chinensis Batal, Fitoterapia, 2014, 100.

44 M. Maiwulanjiang, J. Chen, G. Xin, A. G. Gong, A. Miernisha, C. Y. Du, K. M. Lau, P. S. Lee, J. Chen, T. T. Dong, H. A. Aisa and K. W. Tsim, The volatile oil of Nardostachyos Radix et Rhizoma inhibits the oxidative stress-induced cell injury via reactive oxygen species scavenging and Akt activation in H9c2 cardiomyocyte, J. Ethnopharmacol., 2014, 153(2), 491-498.

45 H. Sun, H. L. Zhang, A. H. Zhang, et al., Network pharmacology combined with functional metabolomics discover bile acid metabolism as a promising target for mirabilite against colorectal cancer, $R S C A d v$., 2018, 8, 30061-30070.

46 H. L. Zhang, A. H. Zhang, X. H. Zhou, et al., High-throughput lipidomics reveal mirabilite regulating lipid metabolism as anticancer therapeutics, RSC Adv., 2018, 8(62), 35600-35610. 
47 H. Sun, H. Wang, A. Zhang, et al., Berberine ameliorates nonbacterial prostatitis via multi-target metabolic network regulation, OMICS: J. Integr. Biol., 2015, 19(3), 186-195.

48 H. Fang, A. H. Zhang, H. Sun, et al., High-throughput metabolomics screen coupled with multivariate statistical analysis identifies therapeutic targets in alcoholic liver disease rats using liquid chromatography-mass spectrometry, J. Chromatogr. B: Anal. Technol. Biomed. Life Sci., 2019, 1109, 112-120.

49 X. Liu, A. Zhang, H. Fang, et al., Serum metabolomics strategy for understanding the therapeutic effects of YinChen-Hao-Tang against Yanghuang syndrome, RSC Adv., 2018, 8, 7403-7413.

50 A. H. Zhang, H. Sun, G. L. Yan, et al., Chinmedomics: A Powerful Approach Integrating Metabolomics with Serum Pharmacochemistry to Evaluate the Efficacy of Traditional Chinese Medicine, Engineering, 2019, 5, 60-68.

51 X. J. Wang, J. L. Ren, A. H. Zhang, et al., Novel applications of mass spectrometry-based metabolomics in herbal medicines and its active ingredients: Current evidence, Mass Spectrom. Rev., 2019, 38(4-5), 380-402.
52 H. Xiong, A. H. Zhang, Q. Q. Zhao, G. L. Yan, H. Sun and X. J. Wang, Discovery and screening quality-marker ingredients of Panax quinquefolius using chinmedomics approach, Phytomedicine, (19), 30097-30102.

53 X. N. Li, A. Zhang, M. Wang, et al., Screening the active compounds of Phellodendri Amurensis cortex for treating prostate cancer by high-throughput chinmedomics, Sci. Rep., 2017, 7, 46234.

54 J. Xie, A. H. Zhang and S. Qiu, Identification of the perturbed metabolic pathways associating with prostate cancer cells and anticancer affects of obacunone, J. Proteomics, 2019, 206, 103447.

55 A. Zhang, H. Wang, H. Sun, et al., Metabolomics strategy reveals therapeutical assessment of limonin on nonbacterial prostatitis, Food Funct., 2015, 6(11), 3540-3549.

56 H. Sun, A. H. Zhang, Q. Song, et al., Functional metabolomics discover pentose and glucuronate interconversion pathways as promising targets for Yang Huang syndrome treatment with Yinchenhao Tang, RSC Adv. , 2018, 8, 36831-36839. 